\title{
Regarding on The Novel Forms of the (3+1) - Dimensional Kadomstev-Petviashvili Equation
}

\author{
Hasan Bulut ${ }^{1, *}$, Betul Demirdag ${ }^{2}$, Haci Mehmet Baskonus ${ }^{3}$ \\ 1,2 Department of Mathematics, University of Firat, Elazig, Turkey \\ ${ }^{3}$ Department of Computer Engineering, University of Munzur, Tunceli, Turkey \\ hbulut@firat.edu.tr, betuldenizd@gmail.com,hmbaskonus@gmail.com
}

\begin{abstract}
This article, we have applied the Bernoulli Sub-Equation method to the (3+1)-Dimensional Kademstev-Petviashvili equation. We have obtained some new analytical solutions such as exponential function and rational solutions by using this technique. We have observed that two analytical solutions have been verified the (3+1)-Dimentional KadomstevPetviashvili equations by using Wolfram Mathematica 9. At the end of this manuscript, we submitted a conclusion in a comprehensive manner.
\end{abstract}

Keywords: Bernoulli Sub-Equation function method; (3+1)-Dimensional Kadomstev-Petviashvili equation; Exponential function solution; rational function solution

\section{Introduction}

With the aid of powerful devices including computer technology and computational in nonlinear sciences, linear and nonlinear equations have been become an important area among scientists and engineers for obtaining various solutions. For example, a solitary wave has been firstly investigated by the Scottish engineer John Russel [1]. He has followed a water wave travelling through a canal. The nonlinear partial differential equation systems give us the better physical explanations of significant mathematical models [2]. Many powerful methods have been developed to find the exact solutions to the NLEEs. Some of these methods such as $\left(G^{\prime} / G\right)$-expansion method $[3,4]$, the improved $\left(\mathrm{G}^{\prime} / \mathrm{G}\right)$-expansion method, the Sumudu transform method [5-6] have been submitted to the literature.

In the rest of this work, we apply the Bernoulli-Sub equation function method (BSFM) to the (3+1)-dimensional Kadomstev-Petviashvili equation [7];

$$
u_{x t}+6 u_{x}^{2}+6 u u_{x x}-u_{x x x x}-u_{y y}-u_{z z}=0 .
$$

\footnotetext{
* Corresponding author: hbulut@,firat.edu.tr
} 
Xie et al. [8] have investigated the some wave solutions of Eq. (1) by the improved tanh function method. We introduce the general properties of BSEFM in section 2. We apply BSEFM in section 3 for obtaining some exponential and rational function solutions. At the end of this study, we submit a conclusion in the comprehensive manner.

\section{Fundamental Properties of Bernoulli Sub-Equation Function Method}

\section{Step 1.}

We consider the following nonlinear partial differential equation (NLPDE) in two variables and a dependent variable $u$;

$$
P\left(u_{x}, u_{t}, u_{x t}, u_{x x}, \cdots\right)=0,
$$

and take the travelling wave transformation

$$
u(x, y, z, t)=U(\eta), \eta=x+y+z-c t,
$$

where $c \neq 0$. Substituting Eq.(3) in Eq.(2) yields a nonlinear ordinary differential equation (NLODE) as following;

$$
N\left(U, U^{\prime}, U^{\prime \prime}, \cdots\right)=0
$$

where

$$
U=U(\eta), U^{\prime}=\frac{d U}{d \eta}, U^{\prime \prime}=\frac{d^{2} U}{d \eta^{2}}, \cdots
$$

\section{Step 2.}

Take trial equation of solution for Eq.(4) as following:

$$
\begin{aligned}
& U(\eta)=\sum_{i=0}^{n} a_{i} F^{i}=a_{0}+a_{1} F+a_{2} F^{2}+\cdots+a_{n} F^{n}, \\
& F^{\prime}=b F+d F^{M}, b \neq 0, d \neq 0, M \in R-\{0,1,2\},
\end{aligned}
$$

where $F(\eta)$ is Bernoulli differential polynomial. Substituting Eq.(5) along with Eq.(6) in Eq.(4), it yields an equation of polynomial $\Omega(F(\eta))$ of $F(\eta)$ as following; $\Omega(F(\eta))=\rho_{s} F(\eta)^{s}+\cdots+\rho_{1} F(\eta)+\rho_{0}=0$.

according to the balance principle, we can obtain a relationship between $\mathrm{n}$ and $\mathrm{M}$.

\section{Step 3.}

Let the coefficients of $\Omega(F(\eta))$ all be zero will yield an algebraic equations system $\rho_{i}=0, i=0, \cdots, s$.

solving this system, we will specify the values of $a_{0}, \cdots, a_{n}$. 


\section{Step 4.}

When we solve Eq.(6), we obtain the following two situation according to $\mathrm{b}$ and d;

$$
\begin{gathered}
F(\eta)=\left[\frac{-d}{b}+\frac{E}{e^{b(M-1) \eta}}\right]^{\frac{1}{1-M}}, b \neq d, \\
F(\eta)=\left[\frac{(E-1)+(E+1) \tanh \left(\frac{b(1-M) \eta}{2}\right)}{1-\tanh \left(\frac{b(1-M) \eta}{2}\right)}\right]^{\frac{1}{1-M}}, b=d, E \in R .
\end{gathered}
$$

Using a complete discrimination system for polynomial, we obtain the solutions to Eq.(4) with the help of Wolfram Mathematica 9 programming and classify the exact solutions to Eq.(4). For a better understanding of results obtained in this way, we can plot two- and three-dimensional surfaces of solutions by taking into consideration suitable values of parameters.

\section{Implementation of the BSEFM}

In this section, we have successfully considered the BSEFM to the $(3+1)$ Dimensional Kademstev-Petviashvili equation for getting some new travelling wave solutions.

Application When we consider the travelling wave transformation and perform the transformation $u(x, y, z, t)=U(\eta), \eta=x+y+z-c t$ which $\mathrm{c}$ is constant non-zero, we obtain NLODE as following;

integrating twice and setting the constants of integration to zero, we obtain $-(c+2) U+3 U^{2}-U^{\prime \prime}=0$.

when we consider to Eq.(5) and Eq.(6) for balance principle between $U^{\prime \prime}$ and $U^{2}$, we obtain the following relationship between $\mathrm{n}$ and $\mathrm{M}$;

$2 M=n+2$.

this relationship gives us some new different analytical solutions for Eq. (1).

\section{Case 1:}

If we take as $n=4$ and $M=3$ in Eq. (11), we can write following equations;

$U=a_{0}+a_{1} F+a_{2} F^{2}+a_{3} F^{3}+a_{4} F^{4}$,

$U^{\prime}=a_{1} b F+a_{1} d F^{3}+2 a_{2} b F^{2}+2 a_{2} d F^{4}+3 a_{3} b F^{3}$

$+3 a_{3} d F^{5}+4 a_{4} b F^{4}+4 a_{4} d F^{6}$,

$U^{\prime \prime}=a_{1} b^{2} F+4 a_{1} b d F^{3}+3 a_{1} d^{2} F^{5}+4 a_{2} b^{2} F^{2}+12 a_{2} b d F^{4}+8 a_{2} d^{2} F^{6}+9 a_{3} b^{2} F^{3}+24 a_{3} b d F^{5}$

$+15 a_{3} d^{2} F^{7}+16 a_{4} b^{2} F^{4}+40 a_{4} b d F^{6}+24 a_{4} d^{2} F^{8}$,

$\vdots$ 
where $a_{4} \neq 0, b \neq 0, c \neq 0, d \neq 0$. When we put Eq. (12-14) in Eq. (10), we obtain a system of algebraic equations. Therefore, we obtain a system of algebraic equations from these coefficients of polynomial of $F$. Solving this system with the help of wolfram Mathematica 9, we find the following coefficients and solutions;

\section{Case 1a.}

For $\mathrm{b} \neq \mathrm{d}$, it can be considered the following coefficients;

$a_{0}=\frac{4 b^{2}}{3}, a_{1}=0, a_{2}=-2 \sqrt{2} b \sqrt{a_{4}}, a_{3}=0, c=-2+4 b^{2}, d=-\frac{\sqrt{a_{4}}}{2 \sqrt{2}}$.

substituting these coefficients in Eq.(12) along with Eq.(9), we obtain the following new exponential function solution for Eq.(1) $u_{1}(x, y, z, t)=\left(\frac{4 b^{2}}{3}-\frac{2 \sqrt{2} b \sqrt{a_{4}}}{e^{-2 b\left(-\left(-2+4 b^{2}\right) t+x+y+z\right)} E E+\frac{\sqrt{a_{4}}}{2 \sqrt{2} b}}+\frac{a_{4}}{\left(e^{-2 b\left(-\left(-2+4 b^{2}\right) t+x+y+z\right)} E E+\frac{\sqrt{a_{4}}}{2 \sqrt{2} b}\right)^{2}}\right)$.
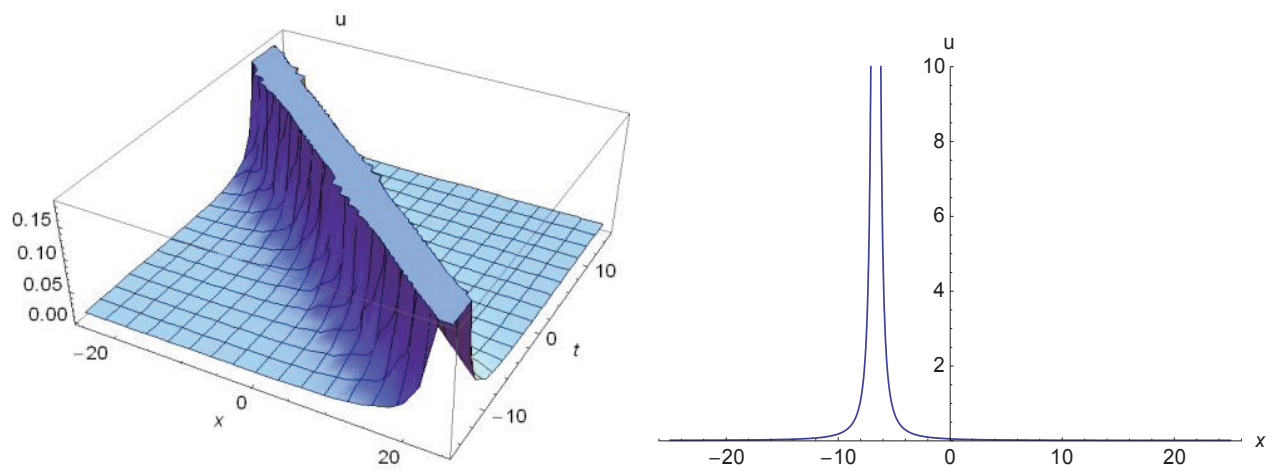

Figure 1. The 2D and 3D surfaces of Eq.(16) under the values of $a_{4}=0.2, b=0.1, E E=-1, y=0.03, z=0.4,-10<t<10,-20<x<20$. and $t=2$ for $2 \mathrm{D}$ surfaces.

\section{Case 1b.}

Another coefficients for Eq.(1) and for $b \neq d$, it can be considered follows $a_{0}=\frac{2+c}{3}, a_{1}=0, a_{2}=-4 d \sqrt{2+c}, a_{3}=0, a_{4}=8 d^{2}, b=-\frac{\sqrt{2+c}}{2}$.

when we regulate Eq.(12) under the terms of Eq.(9,17), we find the other new exponential function 


$$
u_{2}(x, y, z, t)=\left[\frac{4 b^{2}}{3}-\frac{2 b \sqrt{2} \sqrt{a_{4}}}{e^{-2 b\left(-\left(-2+4 b^{2}\right) t+x+y+z\right)} E E+\frac{\sqrt{a_{4}}}{2 \sqrt{2} b}}+\frac{a_{4}}{\left(e^{-2 b\left(-\left(-2+4 b^{2}\right) t+x+y+z\right)} E E+\frac{\sqrt{a_{4}}}{2 \sqrt{2} b}\right)^{2}}\right] .
$$
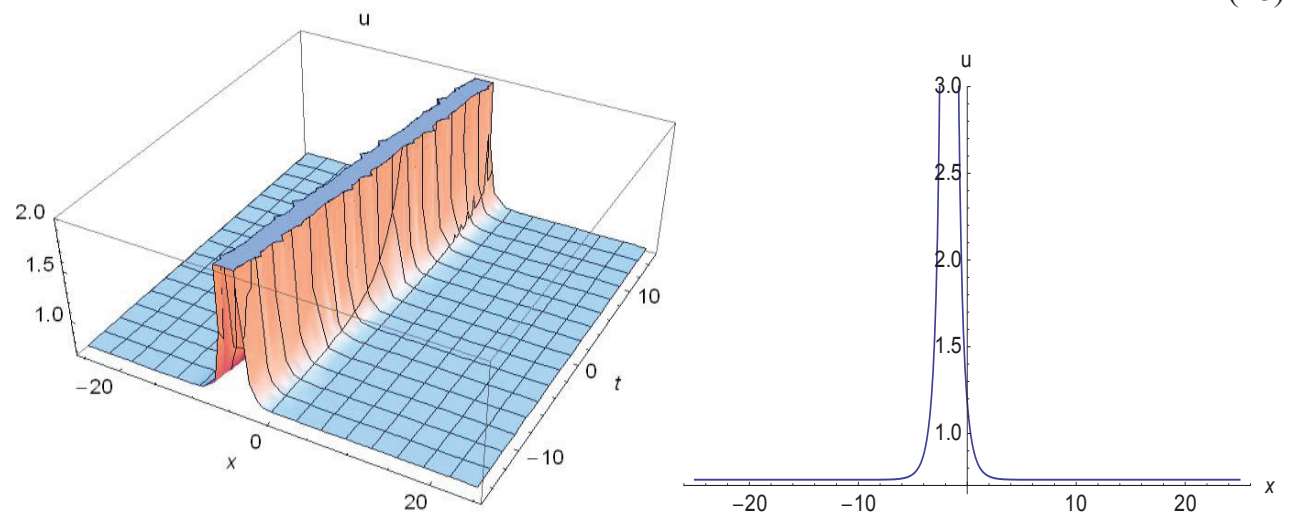

Figure 2. The 2D and 3D surfaces of Eq.(18) under the values of $a_{4}=0.2, E E=-1, b=0.1, y=0.03, z=0.4,-20<x<20,-10<t<10 . t=2$ for $2 \mathrm{D}$ surfaces.

\section{CONCLUSION}

In this paper, we have successfully applied the BSEFM to the Eq. (1). We have obtained some exponential and rational solutions. This method has given a lot of coefficients which gives many results. Some of them have been considered in this paper for obtaining new solutions. If other coefficients is considered, of course, ones can obtain many various solutions for Eq. (1). Therefore, it can be said that this method is a powerful tool for obtaining the solutions of such Eq. (1).

\section{REFERENCES}

1. J.S. Russell, Report on waves, 14th Mtg of the British Association for the Advancement of Science, (1844)

2. C. X. Xue, E. Pan and S. Y. Zhang, Solitary waves in a magneto-electro-elastic circular rod, Smart Materials and Structures, 20(105010), 1-7, (2011)

3. Y.J. Yang, New application of the $\left(G^{\prime} / G, 1 / G\right)$-expansion method to KP equation, Applied Mathematical Sciences, Vol. 7, no. 20, 959-967, (2013)

4. L.X. Li, E.Q. Li and M.L. Wang, the $\left(G^{\prime} / G, 1 / G\right)$-expansion method and its application to traveling wave solutions of the Zakharov equations, Applied Mathematics, Vol. 25, no. 4, 454-462, (2010)

5. F.B.M., Belgacem, 2010. Sumudu transform applications to Bessel functions and equations. Applied Mathematical Sciences 4 (74), 3665-3686.

6. F.B.M. Belgacem, R. Silambarasan, Sumudu transform and trigonometric functions, Journal of Computational and Applied Mathematics, http://dx.doi.org/10.1016/j.cam.2015.12.036, (2016) 
7. Ahmet Bekir, Ferhat Uygun, Exact travelling wave solutions of nonlinear evolution equations by using the $\left(\frac{G^{\prime}}{G}\right)$ - expansion method, Arab Journal of Mathematical Sciences, Vol:18, 73-85, (2012)

8. F.D. Xie, Y. Zhang, Z.S. Lu, Symbolic computation in non-linear evolution equation: application to (3+1)-dimensional Kadomtsev-Petviashvili equation, Chaos, Solitons \& Fractals 24 (2005) 257. 